\title{
Article
}

\section{Persuasive Evidence: Improving Customer Service through Evidence Based Librarianship}

Wendy A. Abbott

Associate Director, Customer Services, Bond University

Gold Coast, Queensland, Australia

E-mail: wendy abbott@bond.edu.au

Received: 10 December 2005

Accepted: 24 February 2006

(C) 2006 Abbott. This is an Open Access article distributed under the terms of the Creative Commons Attribution License (http://creativecommons.org/licenses/by/2.0), which permits unrestricted use, distribution, and reproduction in any medium, provided the original work is properly cited.

\begin{abstract}
Objective - To demonstrate how evidence based practice has contributed to informing decisions and resolving issues of concern in service delivery at Bond University Library.

Methods - The paper critically analyses three evidence based research projects conducted at Bond University Library. Each project combined a range of research methods including surveys, literature reviews and the analysis of internal performance data to find solutions to problems in Library service delivery. The first research project investigated library opening hours and the feasibility of twenty-four hour opening. Another project researched questions about the management of a collection of feature films on DVD and video. The third project investigated issues surrounding the teaching of EndNote to undergraduate students.
\end{abstract}

Results - Despite some deficiencies in the methodologies used, each evidence based research project had positive outcomes. One of the highlights and an essential feature of the process at Bond University Library was the involvement of stakeholders. The ability to build consensus and agree action plans with stakeholders was an important outcome of that process.

Conclusion - Drawing on the experience of these research projects, the paper illustrates the benefits of evidence based information practice to stimulate innovation and improve library services. Librarians, like most professionals, need to continue to develop the skills and a culture to effectively carry out evidence based practice. 


\section{Introduction}

Like most Australian university libraries, Bond University Library employs a range of quality processes and gathers vast quantities of data to measure performance and ensure students and staff receive a high standard of library service. For example, performance indicators such as processing turnaround times are used to verify that resources are available for customers in a timely manner. Usage surveys inform decisions about resource selection and retention and customer surveys objectively measure levels of customer satisfaction and indicate the service areas where further development is needed. These performance indicators and survey results are benchmarked internally over time and externally against other libraries and organisations in the wider community.

A great deal of qualitative data is also gathered to measure the Library's performance including free comments in surveys, feedback received via suggestion boxes (physical and virtual), focus groups, roundtable forums and so on.

While some of these measures are designed to diagnose problems and prescribe the appropriate response or remedial action required, others raise issues for which there is no ready-made solution. At Bond University Library there has been a natural progression from using these systematic quality measures to applying evidence based practice as a rigorous methodology for solving problems in service delivery.

\section{Evidence Based Librarianship in Action}

Despite the absence of an agreed upon definition for evidence based librarianship there is general consensus that evidence based practice in library and information science means applying a sequence of information management processes (Brice, Booth and Bexon 3):
- Specifying the problem

The crucial first stage in evidence based practice is to formulate a focussed, structured, answerable question. Models such as PICO (for evidence based medicine) and SPICE (which is more relevant for information practice) have been developed to help structure the question (Brice, Booth and Bexon 5).

- Finding the evidence to answer the problem

The second stage in evidence based practice is to conduct a comprehensive search of the literature to find evidence relevant to the question posed. Qualitative and quantitative research methods may also be used to locally validate the published evidence (Koufogiannakis and Crumley 124).

- Appraising the evidence The validity, reliability and applicability of the evidence must then be appraised using critical evaluation techniques (Brice, Booth and Bexon 9).

- Applying the results

The results of evidence based research may be directly applicable or may simply improve an understanding of the issues. Variables such as the nature of the user group, timeliness of the evidence, costs, politics and the severity of the problem influence how the results are applied (Koufogiannakis and Crumley 120126).

- Evaluating the outcome

The outcome of evidence based practice can be evaluated on two levels. Firstly on a technical level: have the stages of evidence based practice been successfully followed? Secondly, has the intervention that resulted from the evidence based process actually made the anticipated difference (Booth 127)? 
- Redefining the problem

Through evaluating the outcome of the evidence based practice, the original problem may be redefined or a new question can emerge to guide future research (Booth 128). This last step in the process is sometimes combined with the evaluation stage above.

This paper will describe the application of evidence based practice in solving a number of practical problems that arose at Bond University. Two of the issues emerged from customer feedback and the other evolved through a collaborative project between an academic group and Library staff.

\section{Case Study on Library Opening Hours}

Questions: Should Bond University Library increase opening hours to meet student demand? How realistic is twenty-four hour opening?

While Bond University Library opening hours are good by Australian standards, in 2004 students were increasing pressure on the Library to extend its opening hours including requests for twenty-four hour opening. Requests were received through the Library Suggestion Box, the Rodski Customer Survey and from the Student Council and other student associations.

The decision was made to use an evidence based approach when the issue was escalated to the senior University executive level. Library management decided that a comprehensive report with recommendations based on best practice and other relevant evidence would inform all stakeholders and help to resolve the issue.

The evidence was gathered in a variety of ways:

- The Library's opening hours were compared to those of other Australian university libraries using the Council of Australian University Librarians

(CAUL) annual statistics. This revealed that Bond Library's opening hours were the fourth best in Australia. Only one other institution's opening hours were significantly better.

- A survey of the twenty-four hour facilities of other Australian university libraries was conducted using CAUL to distribute a questionnaire. This showed that six Australian universities had a twenty-four hour facility associated with the Library ranging from a traditional computing laboratory to specially designed and constructed areas incorporating individual and collaborative learning/study facilities. The survey results are available on the CAUL website ("24x7 Library Facilities").

- A review of the literature illustrated that internationally, an increasing number of institutions are opening their libraries for twenty-four hours for some days of the week and new libraries are being built to incorporate a twenty-four hour facility. Research conducted in the United States indicates that students' requests for extended library opening hours are perennial and they vary from requests to open until 2.00 am during exam periods to demands to open twentyfour hours per day seven days of the week, most days of the year (Steele and Walters; Curry; Engel, Womack and Ellis).

- A quantitative analysis of the feedback about opening hours received from Bond University customers in 2004 indicated a low but persistent level of complaints (see Appendix A for more details on how this analysis was performed).

- A quantitative analysis of the usage patterns gathered in the Library's Facilities Use Survey was used to show that the current spread of hours approximated closely to usage 
patterns (see Appendix A for more details on the methodology for the quantitative analysis based on the Facilities Use Survey).

- A detailed costing was prepared for four different scenarios ranging from longer opening hours for the duration of the semester to a short period of twenty-four hour opening leading up to the examination period (see Appendix $B$ for details on how the costing was done).

The evidence was appraised and summarised to produce a report incorporating a short term recommendation to further increase opening hours in the weeks leading up to the examination period. The recommendation for the longer term was to incorporate a twenty-four hour study facility when and if the Library is refurbished. Based on the CAUL survey, such a facility should provide individual and group study spaces, computers, wireless access, laptop ports, photocopier and printing equipment, vending machines, lounges and appropriate security arrangements.

The report was discussed with the Student Council and the senior University executive and while the recommendation for the long term was accepted, the short term proposal was not adopted. Through the process, all stakeholders gained a good understanding of the issues and agreed with the decision that was made.

In the period since the research was undertaken, the concept of refurbishing the Library has progressed and the University has invited proposals from architects to develop a brief for the project. The evidence gathered to resolve the opening hours question has contributed to a better understanding in the wider University community of the Library's role in providing a learning environment in addition to its traditional role as a repository of books and provider of electronic resources. This is helping to convince the senior executive that a refurbishment is necessary to meet student needs.

The refurbishment proposal has defined a new question: "What facilities and services would students like to see included in a refurbished library?" and stakeholder feedback is being gathered using an online survey and focus groups.

\section{Case Study on Management of Access to Feature Films}

Questions: How can the feature films on DVDs and videos be organised and managed to maximise access for the Bond community? Should these resources be more tightly controlled to improve access? What is the most appropriate shelf arrangement?

To support the Bond University School of Film and Television (FTV) programs, the Library has an extensive collection of feature films on DVD and video. The collection benefits the entire Bond community and is heavily used. However, academic staff and students in the FTV School had a number of issues concerning their access to the feature films:

- Due to the popularity of the films, FTV staff and students felt that the DVDs and videos were often not available for their primary purpose (i.e. the teaching and learning needs of FTV courses). They felt that the DVDs and videos they wished to access were often damaged, lost or loaned to borrowers who were not undertaking FTV courses.

- The arrangement of DVDs and videos using the Library of Congress classification and a genre designation was too difficult to use and a title order arrangement was requested.

Evidence to resolve the questions posed was gathered using a survey, analysis of reports of damaged and lost items, analysis of loan patterns and perusal of the policies applied by other university 
libraries. A literature review was also undertaken.

The evidence gathered is summarised as follows:

- A survey of the management practices used in Australian university libraries was conducted using CAUL to distribute a questionnaire. This confirmed that most universities have relatively open access polices for their films on DVD and video; i.e. loans available to all borrowers, material on open access, standard loan periods, multiple items allowed for borrowing.

- The survey revealed libraries were using a variety of security arrangements including traditional Library security strips as well as locked security cases. According to the survey results, most libraries were not experiencing a great deal of theft or loss of feature films. Videos were found to be the most prone to damage due to their aging format.

- The survey showed there were significant differences in how libraries shelve their feature films although about a third of those surveyed use a system that effectively organises them in title order.

- According to the survey, $70 \%$ of CAUL libraries use some form of booking system to ensure films are available for class purposes.

- The results of the CAUL survey are available on the CAUL website ("Feature Film Collection Management").

- The literature review indicated that management of audiovisual collections and especially feature films in academic libraries is an evolving situation for all aspects of their collection management and access. The trend is strongly towards integrating audiovisual collections into the mainstream of library collections, minimising specialist treatment due to the medium (Merry; Brancolini).

- Analysis of Bond Library's loan statistics confirmed that usage of the feature films was high compared with other parts of the collection but that most borrowers were respecting the loan period.

- Analysis of lost and missing reports has been ongoing and this has highlighted the titles that are most susceptible to theft, namely DVDs of popular television series.

Based on these findings FTV academic staff were consulted and a number of steps could be taken immediately. For example, a manual booking system for staff to reserve their titles for class teaching was promoted and an automated system is under consideration. Secondly, feature films were re-shelved in title order. Lastly, security was increased for the popular television series that were found to be susceptible to theft by relocating them to closed access at the Loans Desk. Lockable cases are being investigated in order to be able to return them to open browsing access.

A report with recommendations is being prepared to vary the loans policy, including proposals to limit both the number and length of time that feature films can be borrowed. The new policy is to be discussed and agreed with stakeholders and would incorporate maximum flexibility for academic staff and students in the FTV School to facilitate their borrowing for teaching, learning and research purposes.

\section{Case Study on Teaching Undergraduate Students EndNote}

This case study is at an early stage and thus is less complete than the two case studies described above. 
Question: How can undergraduate students be supported to learn the basics of using EndNote?

At Bond University all undergraduate students are required to undertake a series of core courses including a course delivered by the School of Information Technology (SIT) that is designed to ensure that students understand the key concepts of information technology. The course aims to enable all students to gain competency in using a range of productivity packages. In 2005, it was decided to add EndNote to the list of applications that students would master in this course.

Since the acquisition of a University site licence for EndNote in 2002, the Library has been responsible for support and teaching of the program within the University community. Acknowledging this expertise, SIT staff called on the Library's support in the new initiative.

The literature was reviewed and a survey of practice in Australian universities was conducted via CAUL as summarised below:

- The literature review revealed a plethora of articles about the technical aspects of EndNote including reviews of new versions of the software but relatively few articles on support and teaching EndNote (East; Siegler and Simboli).

- The CAUL survey showed that all but one of the thirty-four universities that responded had a site licence for EndNote and those thirty-three provide support and training in some form. The institutions surveyed indicated that they focus their EndNote training on staff, researchers and postgraduates; however, sixteen provide EndNote training for undergraduates, usually at the request of academic staff.

- In terms of evaluating training, seventeen of the institutions surveyed via CAUL ask participants in workshops to evaluate content and delivery to inform course development but none appeared to assess the learning of participants or use exams to test competency. A few are using quizzes and exercises for learners to check their own progress as they work through EndNote tutorials.

- The results of the survey are available on the CAUL website. ("EndNote")

As the evidence gathered did not reveal any assessment techniques that could be used to evaluate competency in using EndNote, further discussions were held with SIT academic staff and it was agreed that Liaison Librarians would give input to the development of the EndNote mastery tests that would be produced by SIT staff. Library staff conducted EndNote training for the SIT tutors who would deliver the practical tutorials for undergraduates. It was also agreed that a Liaison Librarian would be coopted to introduce EndNote in the lecture program.

At the end of the semester, students' results on the EndNote mastery test will be analysed. A broader question about the impact of teaching EndNote still needs to be researched and answered: How will teaching undergraduate students EndNote impact on their overall information skills?

\section{Evaluating Our Performance}

Using evidence based practice at Bond University Library constitutes a deliberate strategy for introducing change and continuous improvement. While professional experience and judgement are not discounted, using evidence based practice is an attempt to move away from relying solely on anecdote and personal experience to solve important problems in service delivery.

Analysing our performance in applying evidence based practice at Bond University illustrates our successes as well 
as our limitations. One of the highlights and an essential feature of the process at Bond Library is the involvement of stakeholders. This varies from seeking their input to partnering with them to learn from their expertise. The ability to build consensus and agree upon action plans with stakeholders is an important outcome of that process. Stakeholder involvement is akin to the patient centred approach that is a hallmark of evidence based medicine (Sackett et al.). In the Bond context, it continues a strong tradition of developing and maintaining good relationships with customers.

In critically analysing each step of the evidence based approach we used, the three case studies have been a valuable learning experience:

Specifying the question: In the case of the opening hours issue and the access to feature films problem, the questions were focussed by analysing the feedback from customers. In the case of support for EndNote the question was not as easy to define and as it transpired, the broader question that could have been asked still needs to be addressed. In all the projects a structured process such as PICO or SPICE could have helped focus the questions and in turn would have aided the process of choosing the research design (Brice, Booth and Bexon 5-6).

Finding the evidence to answer the problem: In each case, a range of techniques was used to gather evidence, including literature searches, surveys and other data gathering methods. As reported by Brice, Booth and Bexon (7), the literature search process was complicated due to the range of information sources that comprise the evidence base. The databases we searched included Ebsco's Academic Search Premier, Business Source Premier, Library Information Science and Technology Abstracts and Professional Development Collection. InfoTrac Expanded Academic and ERIC were also searched. In each case, structured search methods were used across the target databases. Searching a wider range of sources could have produced better results but this was not practical within the time available.

In each case, a survey was conducted of CAUL members (40 Australian university libraries) and the Council of New Zealand University Librarians members (6 New Zealand university libraries). The surveys gathered a broad base of valid and reliable evidence about current practice in the sector. However, the survey methodology we used could have been more rigorous. For example, the questions could have been more thoroughly tested to obtain more accurate and comprehensive information and more thought could have been given to how the responses would be analysed. If the survey methodology had been automated, the results would have been easier to analyse. Web-based survey applications with inbuilt analysis methodologies can save a lot of time for those responding to surveys as well as those analysing the responses. While using surveys as the predominant research method may not meet the gold standard for research rigor (West 12-13), in the case of the questions raised at Bond University, they satisfied the need to gather evidence quickly so that pressing service problems could be resolved in a timely manner.

Appraising the evidence: The evidence base generated by the literature searches was informative and contributed to an improved understanding of the issues. But as found by Booth and Brice (10), the results for the literature searches conducted for all three case studies showed few studies with findings that could be transferred to the current situations. This may be inevitable given the practical nature of the questions being posed. Fortunately, the lack of transferable results in published studies was partly overcome by the broad base of evidence generated through the CAUL surveys. The survey information was analysed and the major trends for the sector could be readily identified and used as a guide for practice at Bond University. Another valuable evidence base in two of the three cases described was the analysis of local usage statistics. 
Applying the results: In determining the applicability of the evidence assembled, questions about costs, severity, timeliness and politics, as suggested by

Koufogiannakis and Crumley (121-123) all came into play. In each case feedback from stakeholders had been significant in raising the issues in the first instance. Their involvement was equally important in deciding how the results of the research would be applied to address those issues. In each case, library staff discussed the evidence with stakeholders and through the process a consensus developed on how best to proceed. Both library staff and stakeholders were informed by the research and assured that plans to take action were soundly based.

Evaluating the outcome and redefining the problem: The opening hours case was concluded to the satisfaction of both library staff and stakeholders and is now pending a refurbishment of the Library. This has effectively defined a new direction for our research. The other two cases have not yet been concluded although following initial research and consultation with stakeholders some interim actions have been undertaken and this has allayed some of the biggest concerns of stakeholders especially in the case of managing access to feature films. In evaluating the EndNote case study, the broader question about the impact of teaching EndNote on students' information skills has emerged to define a new research question.

\section{Conclusion}

The three case studies illustrate Bond Library's first intentional foray into using evidence based practice to capitalise on well established quality processes and to stimulate innovation and change. The concept of "professional practice built on the explicit and judicious use of current best research findings in making decisions about the performance of the day-to-day role" seems hard to fault (Todd 9). However, as the Bond University experience has shown, there are barriers to overcome even when the work environment is conducive. Like most professionals, librarians at Bond University need to develop the skills and a culture to effectively carry out evidence based practice. These include the skills to articulate questions, undertake research, appraise research findings and implement a course of action. Above all it requires librarians to develop a culture of questioning and reflecting on what we do. Despite the limitations in some aspects of the evidence based practice used at Bond Library, the success of the three research projects described in this paper amply demonstrates the benefits of an evidence based approach. Persuasive evidence!

\section{Works Cited}

“24x7 Library Facilities.” Surveys/Questionnaires Distributed to CAUL No. 2004/27. CAUL. 17

February 2006

$<$ http://www.caul.edu.au/surveys/>.

Booth, Andrew. "Evaluating Your

Performance." Evidence-Based

Practice for Information Professionals: A Handbook. Ed. Andrew Booth and Anne Brice. London: Facet Publishing, 2004. 127-37.

Booth, Andrew, and Anne Brice. "Why

Evidence-Based Information

Practice?" Evidence-Based Practice for Information Professionals: A Handbook. Ed. Andrew Booth and Anne Brice. London: Facet Publishing, 2004. 1-12.

Brancolini, Kristine R. "Video Collections in Academic Libraries." Video Collection Development in MultiType Libraries: A Handbook. Ed. Gary P. Handman. Westport, CT: Greenwood Press, 2002. 47-75.

Brice, Anne, Andrew Booth and Nicola Bexon. "Evidence Based Librarianship: A Case Study in the Social Sciences." Proceedings of the 71st IFLA General Congress and Council, August 14-18, 2005, Oslo, Norway. 19p. IFLA. 1 Sept. 2005, 
$<$ http://www.ifla.org/IV/ifla71/papers

111e-Brice Booth Bexon.pdf $>$.

Curry, Ann. “Opening Hours: The Contest between Diminishing Resources and a 24/7 World." Journal of Academic Librarianship 29 (2003): 375-85.

East, John W. "Academic Libraries and the Provision of Support for Users of Personal Bibliographic Software: A Survey of Australian Experience with Endnote." LASIE 32.1 (2001): 64-70.

"EndNote." Surveys/Questionnaires Distributed to CAUL No. 2005/4. CAUL. 17 February 2006 $<$ http://www.caul.edu.au/surveys/>.

Engel, Debra, Kay Womack and Ursula Ellis. "Opening a Library Twenty-Four Hours." Journal of Library Administration 36 (2002): 95-109.

"Feature Film Collection Management" Surveys/Questionnaires Distributed to CAUL No.2005/8. CAUL. 17 February 2006

$<$ http://www.caul.edu.au/surveys/>.

Koufogiannakis, Denise, and Ellen Crumley. "Applying Evidence to Your Everyday Practice." Evidence-Based Practice for Information Professionals: A Handbook. Ed. Andrew Booth and Anne Brice. London: Facet Publishing, 2004. 119-126.

Merry, Lois K. "The Devil in the Details: An Academic Library Acquires a Video Collection." Library Collections, Acquisitions and Technical Services 28 (2004): 298-311.

Sackett, David L., et al. Evidence-Based Medicine: What It Is and What It Isn't. 1996. Centre for Evidence-Based Medicine. 7 Sept. 2005 $<$ http://www.cebm.net/ebm is isnt.as $\mathrm{p}>$.

Siegler, Sharon, and Brian Simboli. "EndNote at Lehigh." Issues in Science and Technology Librarianship 34
(2002). Association of College and Research Libraries, Science and Technology Section. 1 Sept. 2005 $<$ http://www.istl.org/02spring/article4.html .

Steele, Patricia Ann, and Carolyn Walters. Extended Library Hours, SPEC Kit no. 264. Washington, D.C.: Association of Research Libraries, 2001.

Todd, Ross. "Ross Todd's Letter from America." Orana 39.3 (2003): 9-10.

West, Kathy. "The Librarianship Report: Convincing Evidence; Seeking Out the Best Available Evidence." Information Outlook 7 (2003): 12-15. 


\section{Appendix A: Details on Quantitative Analyses Used}

\section{Quantitative Analysis of Feedback about Opening Hours}

To assess the level of feedback about opening hours, unsolicited feedback received in the Library's Suggestion Box and "free" comments recorded in the Library's 2004 Customer Survey (conducted by the Rodski Behavioural Research Group) were measured as a percentage of the total number of comments received.

The analysis showed that in 2004, $7 \%$ of Suggestion Box comments and almost $4 \%$ of free comments in the customer satisfaction survey were requests for longer opening hours. In the first half of 2005 the number of Suggestion Box requests for longer opening hours grew to $10 \%$.

\section{Usage patterns identified in the Facilities Use Survey}

Bond University Library regularly conducts a Facilities Use Survey by counting the number of users occupying the Library's various facilities (study carrels, open tables, lounge chairs, Library catalogues, student use computers, laptop ports, group study rooms, DVD and video players, etc.). The survey also includes a total head count. A precise count is taken four times daily throughout the Library's opening hours during the survey period (two weeks each semester).

In 2004, the Facilities Use Survey illustrated that patronage was heaviest at mid afternoon and showed that patronage grew and declined on either side of the peak time of day; building gradually from opening time and tailing off towards closing time. 


\section{Appendix B: Detailed Costing to Extend Library Opening Hours}

The major expense in extending the Library's opening hours is the cost of additional staffing and to illustrate the scale of funding that would be required, the costs of four scenarios were developed. The scenarios are listed below from the most expensive (1.) to the least expensive (4.) diminishing by approximately $50 \%$ for each option down the list.

1. Open $24 \times 7$ for 2 weeks per semester during exam periods: cost per annum for 2 libraries (Main and Law)

2. Open extended hours for Friday, Saturday and Sunday (until 9.00pm) throughout the semester: cost per annum for 2 libraries (Main and Law)

3. Open until midnight (Monday to Thursday) for second half of semester (7 weeks): cost per annum for 2 libraries (Main and Law)

4. Extend opening hours for Friday, Saturday and Sunday until $11.00 \mathrm{pm}$ for the last three weeks of the semester: cost per annum for 2 libraries (Main and Law).

Option 1 would also require additional security arrangements including CCTV and close surveillance by Security staff. 